\title{
Congenital renal anomaly in a patient with situs inversus
}

\section{P Chaturvedi, K Thomas}

Answers on p 359.

$\Lambda$ 65 year old man was referred to the urology clinic with a two month history of right loin pain. He had no previous urological history. Situs inversus had been diagnosed previously during a routine appendicectomy.

Clinical examination and blood pressure were normal. Laboratory evaluation including blood urea, serum creatinine, 24 hour urinary protein, and urine culture were normal. A plain abdominal radiograph showed no calculi. An ultrasound scan identified a suspicious mass lesion, which demonstrated increased vascularity in the lower pole of the left kidney. A subsequent computed tomogram failed to identify a mass lesion but did reveal a congenital abnormality (fig l) in addition to the previously diagnosed situs inversus (fig 2). The patient was reassured and discharged with instructions for a regular blood and urine check by his family doctor.

\section{QUESTIONS}

(1) Name the congenital renal anomaly identified in fig 1 .

(2) What is the incidence and sex ratio of this anomaly?

(3) What complications occur with this condition?

(4) Name three other genitourinary anomalies that can be associated with this condition.

Postgrad Med J 2003;79:355

\section{Authors' affiliations}

P Chaturvedi, K Thomas, Ysbyty Gwynedd North West Wales NHS Trust, Bangor LL57 2PW, UK

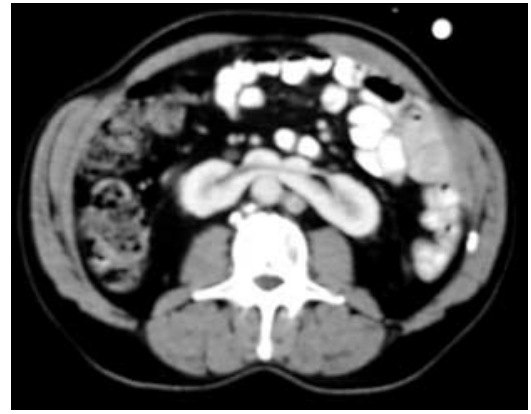

Figure 1 Computed tomogram showing congenital abnormality.

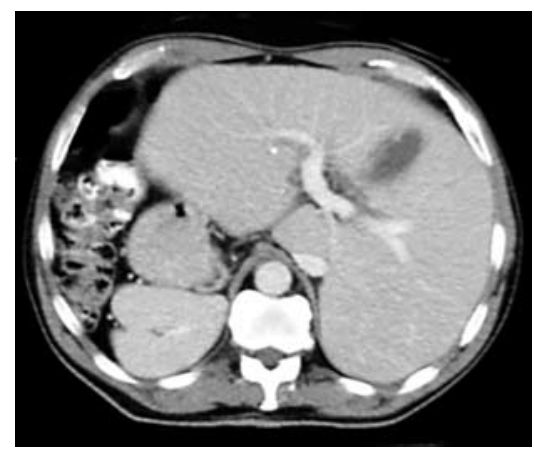

Figure 2 Computed tomogram showing situs inversus.

Correspondence to: $\mathrm{Mr}$ Chaturvedi patanjali_c@hotmail.com

Submitted 10 November 2002

Accepted 24 January 2003
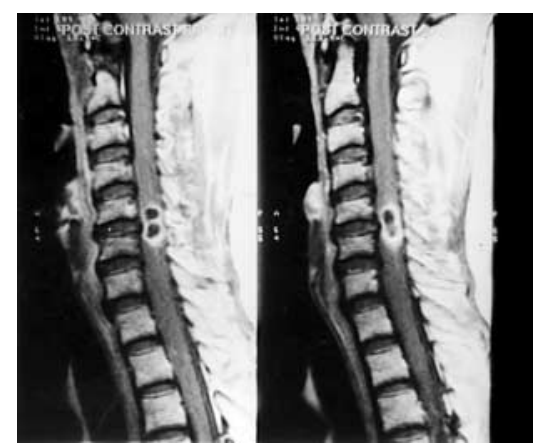

Figure 1 Sagittal MRI scan of the spine showing a hypointense cyst at C5-6.

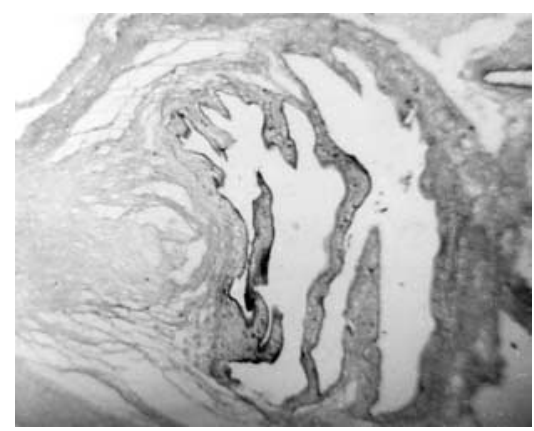

Figure 2 Cysticercus cyst with scolex (larval cyst; haematoxylin and eosin $\times 100$ ). of the cardiovascular, respiratory or gastroenterological systems. Neurological examination showed normal higher function and no abnormality of cranial nerves. Motor system examination revealed atrophy of small muscles of the hand in the left upper limb, tone was increased in all four limbs, while power was more decreased in the lower limbs (grade III) when compared with the upper limbs (grade IV). Sensory system examination revealed a decrease in sensations with upper level at the C4 dermatome (posterior column was more affected than the spinothalamic). On examination of the reflexes, the biceps was absent while the supinator/triceps was increased in the left upper limb as were reflexes in the other three limbs. 
Abdominal reflex was absent whereas the plantar was bilaterally extensor. Computed tomography of the head revealed normal findings. Magnetic resonance imaging (MRI) of the spine (fig 1) showed a low intensity lesion at C5-6 with a slightly hyperintense nodule on TIWl and high intensity lesion on T2Wl with widened cord. Laminectomy was performed and the lesion removed was sent for histopathology (fig 2).

\section{QUESTIONS}

(1) What is the diagnosis?

(2) What are the treatment options?

Postgrad Med J 2003;79:355-356

Authors' affiliations

N K Sharma, Department of Neurosurgery, Pt B D Sharma Postgraduate Institute of Medical Sciences, Rohtak (Haryana), India

\section{An interesting case of hemiparesis}

\section{S Arora, A Arora, R P S Makkar, A Monga}

\section{Answers on p 359.}

A 40 year old man was brought to the hospital with a history of pain and progressive diminution of vision in his left eye for one month, weakness of left half of body for 15 days, and altered sensorium for five days. There was no history of fever, cough, headache, vomiting, seizures, or trauma to the head. The relatives denied any history of sexual contact outside marriage, intravenous drug abuse, or recent travel by the patient. There was no significant illness in the past, including tuberculosis. On examination, the patient was conscious but drowsy and disoriented. His pulse rate was 90 beats/min, blood pressure was $130 / 80 \mathrm{~mm} \mathrm{Hg}$, and respiratory rate was 20 breaths/min. General physical

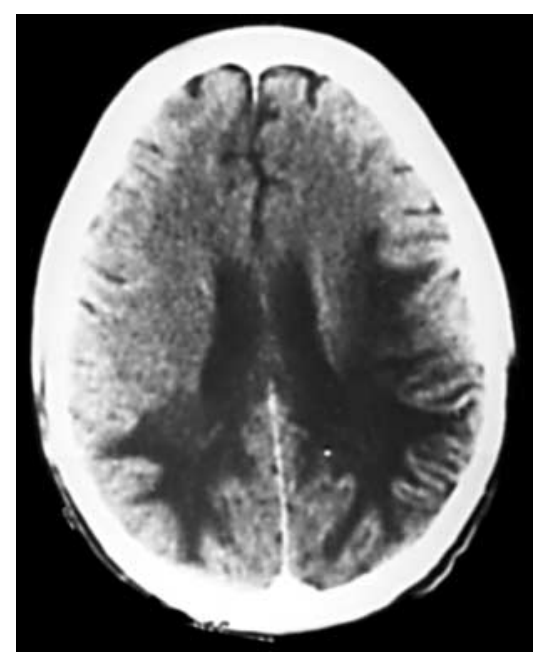

Figure 1 Contrast enhanced computed tomogram of head. examination and systemic examination of chest, cardiovascular system, and abdomen was normal. Neurological examination revealed left sided hemiparesis and left upper motor neurone facial palsy. There were no meningeal signs and fundus examination showed
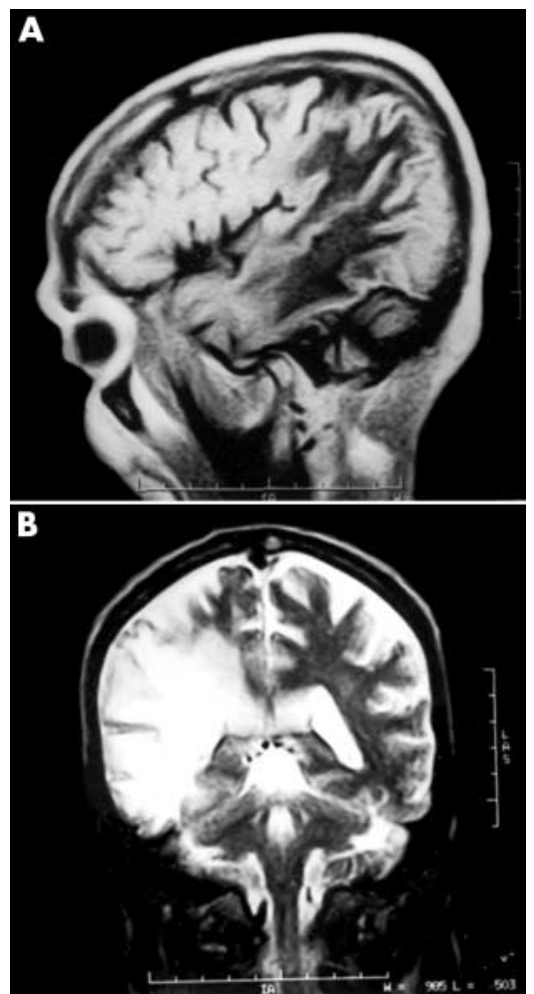

Figure 2 MRI of head: (A) T1 weighted sagittal image and (B) T2 weighted coronal image.
T S Jaswal, S Singh, N Marwah, Department of Pathology

H Singh, M Singh, Department of Medicine

Correspondence to: Dr Sunita Singh, 881/23, DLF Colony, Rohtak-124001 (Haryana), India; hps $1 @ v s n l . n e t$

Submitted 11 June 2002

Accepted 25 November 2002

pallor of the left optic disc suggestive of optic atrophy.

Initial laboratory investigations revealed a haemoglobin concentration of $99 \mathrm{~g} / \mathrm{l}$, total leucocyte count of $5.6 \times 10^{9} / \mathrm{l}$, with a normal differential count. Other routine investigations including blood glucose, liver and kidney functions, urine examination, chest radiography, and electrocardiography were normal. Cerebrospinal fluid examination revealed 5 lymphocytes/high power field with normal proteins and glucose levels. No acid fast bacilli, fungus, or malignant cells were seen and polymerase chain reaction test for Mycobacterium tuberculosis was negative. Computed tomography and magnetic resonance imaging (MRI) of the head are as seen in figs 1 and 2 respectively. Enzyme linked immunosorbent assay (ELISA) for HIV was reactive and western blot was confirmatory

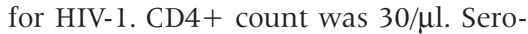
logical tests for syphilis were nonreactive. Stereotactic brain biopsy was suggested but was refused by the patient's family. Highly active antiretroviral therapy (HAART) was started but the patient continued to deteriorate and died on the fifth day of admission.

\section{QUESTIONS}

(1) What is the differential diagnosis in this patient?

(2) What are the computed tomography and MRI findings?

(3) How is the diagnosis confirmed?

Postgrad Med J 2003;79:356

\section{Authors' affiliations}

S Arora, A Arora, R P S Makkar, A Monga

Department of Medicine, Sitaram Bhartia

Institute of Science and Research, New Delhi, India

Correspondence to: Dr Ravinder P S Makkar, Department of Medicine, Sitaram Bhartia Institute of Science and Research, B-16, Mehrauli Institutional Area, New Delhi, India; makkar_r@yahoo.com

Submitted 29 November 2002

Accepted 23 December 2002 